\title{
Minute Unit of Plane Angle
}

National Cancer Institute

\section{Source}

National Cancer Institute. Minute Unit of Plane Angle. NCI Thesaurus. Code C68860.

A unit of angular measure equal to $1 / 60$ degree or to 60 arcseconds. This unit is often called the arcminute to distinguish it from the minute of time. There are 21600 arcminutes in a circle. The international standard ISO 31 requires that angles be stated in degrees and decimal fractions of the degree, without use of arcminutes and arcseconds. 